\title{
Foreword: The Song of Silence
}

Most readers will be familiar with sheet music, the written form of the notes a musician plays. Written music has an interesting set of notations that does not have an analogous item in standard written text dedicated to silences or moments of rest. The musical notes to be played are shown on the staff alongside the prescribed silences, and the silences can be as complex as the notes themselves. Speech and music performance share a number of similarities, including that both are exquisite in the precision of their timing, especially among the silences. Composers write silences into the music very carefully, and musicians use those silences for a variety of artistic effects. Perhaps it is time we devoted more attention to how communicating with language artfully uses silences. The volume you are about to read is filled with observations about silence and its meanings.

Compared with studying spoken words, test scores or written text, at first glance studying silence might appear as if looking for something occult - not in the supernatural sense of the word but as something hidden from view. Yet a thoughtful approach to silence suggests that it is far more than simply the absence of speech. Silence itself is meaningful and communicative. Parables, expressions and well known quotations suggest that the persons wise among us have something to say about silence. First among them in my view - my mother told me to say nothing if I had nothing good to say, and that has proven to be sage advice. Searching the web for what thoughtful people had to say about silence produces a plethora of observations concerning the value and meaning of silence. Across the many inspiring quotes that are available there are at least two general themes.

The first theme is that silence communicates something meaningful. Silence is not merely the absence of speech: silence itself says something. Psychology Today has a webpage of quotes devoted to silence (see Sarkis, 2013). Among them is 'He who does not know how to be silent will not know how to speak' (Ausonius). Keywords used in other quotes to describe the positive side of silence include 'golden', 'perfect', 'strong', 'safe' and 'peaceful'. In classrooms of all types, teaching and learning require the active balancing of talking and silence across the whole group. Silence 
provides the necessary boundary for each person's meaningful speech. Without silences, the talk in a classroom would be a virtually incoherent wall of sound. Silence can be a most welcome thing.

The second theme among the quotes is more subtle and troublesome. A number of authors have suggested that silence is problematic when it is born out of fear and avoidance, as in a context where one could/should be speaking out about injustice in the world. Psychology Today quotes Martin Luther King Jr as saying 'In the end, we will remember not the words of our enemies, but the silence of our friends'. A reluctance to speak based on fear or apprehension is restrictive and at times can be quite debilitating. Language teachers might be especially troubled if a class is too reticent to speak when required. The silent learners who fear being less than perfect or losing face are depriving themselves of important learning opportunities, especially in the pursuit of language learning. Reticence based on fear is among the many reasons for silence, an unwelcome state with potentially enduring consequences.

The editors of this volume, King and Harumi, along with the contributors, deserve to have their work carefully considered. Applied linguistics, and studies of communication more generally, seem to have under-appreciated the many ways in which silence emerges in language learning and use. Although this volume contextualises the study of silence in East Asia, where the cultural dimensions of the work on silence have been a focus often attributed to influence by Confucian philosophy, the complex, interacting processes that dynamically create silence certainly extend beyond East Asian locations and Confucian philosophy. If silence is an inherently communicative, multidimensional phenomenon with nuanced meaning, it is so everywhere. The stereotype of Western classrooms being talkative, especially in contrast to East Asian classrooms, is a belief that needs to be examined more closely so that the meanings of silence can be better understood outside the Asian context. This volume contributes a necessary perspective to that pursuit. Language teachers around the world must deal with issues of talking and silence where different contexts will accentuate different processes. We can work towards identifying the commonalities among those processes within and across cultures, education systems, schools, classrooms and persons.

Thinking about silence in the context of individuals and their personality traits suggests the possibility of happy apples and anxious oranges. Some people talk more than others and some people are more comfortable with silence than others. The happy apples may be using silence to create a comfort zone for reading or thoughtful contemplation, or as an escape from a noisy world; for many people, silence is a welcome respite. For anxious oranges, silences are uncomfortable states to be avoided, and as the length of silence increases so does the perceived stress. When silence arises out of fear, anxiety, perfectionism, hesitations and so on, the teaching and learning process is disrupted. Conventional wisdom suggests 
that, in general, we should avoid comparing apples and oranges. The themes of meaningful versus avoidant silences identified above suggest some value in separating the positive and negative assumptions about silence, to avoid comparing the happy apples and anxious oranges. The patterns blending talk and silence in pleasant or unpleasant ways extend from the intrapersonal to intercultural relationships.

Perhaps we should take it as given from here on that silence - at its core - is an adaptive response constructed within context in a meaningful way. Taking silence as an active response, rather than the absence of an active response (talking), may lead to innovative approaches to creating the desired balance between the sound and the silence in the language classroom. Whereas such a balance has been coded explicitly into music, perhaps the present volume will remind teachers, learners and researchers to be more sensitive to the multiple, deliberate, adaptive and sometimes artistic purposes of silence.

Peter MacIntyre

Cape Breton University, Canada

\section{Reference}

Sarkis, S.A. (2013) 20 quotes on silence. Psychology Today. At https://www.psychologytoday. $\mathrm{com} / \mathrm{gb} / \mathrm{blog} /$ here-there-and-everywhere/201312/20-quotes-silence (last accessed February 2020). 
